\title{
Search for the strong pair production of squarks and gluinos in events with an isolated lepton, jets and missing transverse momentum at $\sqrt{s}=13 \mathrm{TeV}$ with the ATLAS detector
}

Andrei Alexandru Geanta*, on behalf of the ATLAS Collaboration

IFIN-HH Bucharest (RO)

E-mail: andrei.alexandru.geanta@cern.ch

\begin{abstract}
This document presents the search for the strong pair production of squarks and gluinos in events with one isolated lepton, jets and missing transverse momentum in the final state. In this analysis, strongly interacting supersymmetric particles are assumed to decay into charginos $\tilde{\chi}_{1}^{ \pm}$and light quarks, and each chargino subsequently decays into a $W$ boson and the lightest neutralino $\tilde{\chi}_{1}^{0}$. The analyzed data recorded by the ATLAS detector at the Large Hadron Collider (LHC) from 2015 to 2016 corresponds to a total integrated luminosity of $36.1 \mathrm{fb}^{-1}$ of proton-proton collisions at $\sqrt{s}=$ $13 \mathrm{TeV}$. The general description of the targeted signal models, expected background processes and an overview of the analysis strategy are shown. In the context of this model, a statistical interpretation of the observed data is provided and the exclusion limits on the squarks/gluinos, chargino and lightest neutralino masses are illustrated in two benchmark scenarios.
\end{abstract}

European Physical Society Conference on High Energy Physics - EPS-HEP2019 -

10-17 July, 2019

Ghent, Belgium

${ }^{*}$ Speaker. 


\section{Introduction}

Supersymmetry (SUSY) is a theoretical extension of the Standard Model (SM) which predicts that for each SM particle there is a corresponding supersymmetric partner. In R-parity-conserving SUSY scenarios, supersymmetric particles are produced in pairs at the LHC and decay into SM particles and the lightest supersymmetric particle (LSP), which is stable and escapes detection. In the following, the results of a search for gluino/squark pair production in final states with one isolated lepton (electron or muon), missing transverse energy and multiple jets using proton-proton collision data recorded by the ATLAS detector [1] is presented. The results of this search have been published in [2]. The decay modes of the two simplified SUSY models considered in this search are shown in Figure 1. In the context of these models, the branching ratios of the decay of interest are assumed to be $100 \%$. Two benchmark scenarios are considered in order to probe a broad range of SUSY mass spectra. In one benchmark scenario, $m_{\tilde{\chi}_{1}^{0}}$ is set to $60 \mathrm{GeV}$, while the $m_{\tilde{g} / \tilde{q}}$ and the relative mass splitting $x \equiv \frac{m_{\tilde{\chi}_{1}^{ \pm}}-m_{\tilde{\chi}_{1}^{0}}}{m_{\tilde{g} / \tilde{q}}-m_{\tilde{\chi}_{1}^{0}}^{0}}$ are free parameters. In the other benchmark scenario, the chargino mass is set to be exactly halfway between the masses of the gluino/squark and the LSP by requiring $x=\frac{1}{2}$, while $m_{\tilde{g} / \tilde{q}}$ and $m_{\tilde{\chi}_{1}^{0}}$ are free parameters.

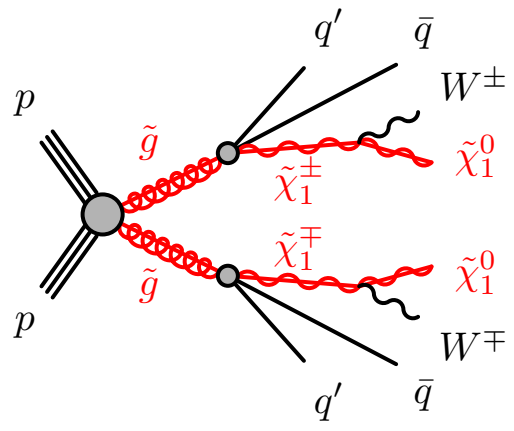

(a) gluino pair-production

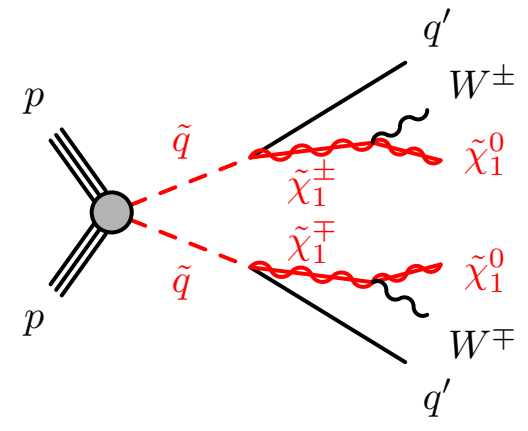

(b) squark pair-production

Figure 1: The decay modes of gluinos (a) and squarks (b) in the simplified signal models considered in this search.

\section{Analysis strategy}

\subsection{Signal Regions}

Four signal regions (SRs) requiring different jet multiplicities are designed to cover a wide range of mass spectra. Table 1 presents the four signal regions (SRs) designed for the gluino/squark models. The "two-jets" (2J) signal region targets compressed scenarios $\left(m_{\tilde{g} / \tilde{q}} \approx m_{\tilde{\chi}_{1}^{ \pm}} \approx m_{\tilde{\chi}_{1}^{0}}\right)$ where most of the decay products tend to have a low $p_{\mathrm{T}}$. Electrons (muons) are required to have a minimum $p_{\mathrm{T}}$ of $7(6) \mathrm{GeV}$, and the maximum $p_{\mathrm{T}}$ of $\min \left(5 \cdot N_{\mathrm{jet}}, 35\right) \mathrm{GeV}$. The upper cut on the lepton $p_{\mathrm{T}}$ ensures the orthogonality of the $2 \mathrm{~J} \mathrm{SR}$ to the other regions. The "four-jets" (4J) signal regions are designed for the variable- $x$ models. The $4 \mathrm{~J}$ high- $x$ SR targets scenarios where $m_{\tilde{\chi}_{1}^{ \pm}} \approx m_{\tilde{g} / \tilde{q}}$, in which the $W$ boson resulting from the $\tilde{\chi}_{1}^{ \pm}$decay is highly energetic, leading to a high $p_{\mathrm{T}}$ lepton. The 
$4 \mathrm{~J}$ low- $x$ SR targets scenarios where $m_{\tilde{\chi}_{1}^{ \pm}} \approx m_{\tilde{\chi}_{1}^{0}}$. Due to the large mass splitting between $m_{\tilde{g} / \tilde{q}}$ and $m_{\tilde{\chi}_{1}^{ \pm}}$the jets are expected to be highly energetic, leading to higher effective mass ( $\left.m_{\text {eff }}\right)$ and large aplanarity values than in high- $x$ region. An upper cut on the transverse mass $\left(m_{\mathrm{T}}\right)$ is used to ensure the orthogonality between the 4J SRs. The "six-jets" (6J) signal region targets scenarios with high gluino/squark mass. Events with one high $p_{\mathrm{T}}$ lepton and at least six jets are required to keep the orthogonality with the $2 \mathrm{~J}$ and $4 \mathrm{~J}$ SRs. Additionally, each SR is binned using three/four $m_{\text {eff }}$ bins, and is split into two categories: bTag, requiring at least one b-tagged jet, and bVeto, requiring no b-tagged jets.

\begin{tabular}{|c|c|c|c|c|}
\hline SR & $2 J$ & 4J high- $x$ & 4J low- $x$ & $6 \mathrm{~J}$ \\
\hline $\begin{array}{l}N_{\ell} \\
p_{\mathrm{T}}^{\ell}[\mathrm{GeV}]\end{array}$ & $\begin{array}{c}=1 \\
>7(6) \text { for } e(\mu) \text { and } \\
<\min \left(5 \cdot N_{\mathrm{jet}}, 35\right)\end{array}$ & $\begin{array}{c}=1 \\
>35\end{array}$ & $\begin{array}{c}=1 \\
>35\end{array}$ & $\begin{array}{c}=1 \\
>35\end{array}$ \\
\hline$N_{\text {jet }}$ & $\geq 2$ & $4-5$ & $4-5$ & $\geq 6$ \\
\hline $\begin{array}{l}E_{\mathrm{T}}^{\mathrm{miss}}[\mathrm{GeV}] \\
m_{\mathrm{T}}[\mathrm{GeV}] \\
\text { Aplanarity } \\
E_{\mathrm{T}}^{\text {miss }} / m_{\mathrm{eff}} \\
\end{array}$ & $\begin{array}{c}>430 \\
>100 \\
\quad \ldots \\
>0.25 \\
\end{array}$ & $\begin{array}{l}>300 \\
>450 \\
>0.01 \\
>0.25\end{array}$ & $\begin{array}{c}>250 \\
150-450 \\
>0.05 \\
\cdots \\
\end{array}$ & $\begin{array}{l}>350 \\
>175 \\
>0.06 \\
\quad \cdots \\
\end{array}$ \\
\hline $\begin{array}{l}N_{\mathrm{b}-\mathrm{jet}}(\mathrm{excl}) \\
m_{\text {eff }}[\mathrm{GeV}](\mathrm{excl}) \\
m_{\text {eff }}[\mathrm{GeV}](\text { disc })\end{array}$ & $\begin{array}{c}3 \text { bins } \in[700,1900] \\
+[>1900] \\
>1100\end{array}$ & $\begin{array}{c}=0 \text { for } b \text {-veto, } \geq \\
2 \text { bins } \in[1000,2000] \\
+[>2000] \\
>1500\end{array}$ & $\begin{array}{l}\text { for } b \text {-tag } \\
\begin{array}{l}2 \text { bins } \in[1300,2000] \\
\quad+[>2000] \\
\quad>1650(1300) \\
\text { for gluino (squark) }\end{array}\end{array}$ & $\begin{array}{c}3 \text { bins } \in[700,2300] \\
\quad+[>2300] \\
>2300(1233) \\
\text { for gluino (squark) }\end{array}$ \\
\hline
\end{tabular}

Table 1: Overview of the selection cuts for the SRs used for gluino/squark simplified models [2].

\subsection{Background estimation}

The dominant SM background contributions originate from top quark ( $t \bar{t}$ and single top) and $W+$ jets production. The minor backgrounds originate from the production of $Z+j e t s, t \bar{t}+V$ (a pair of top quarks in association with a $W$ or a $Z$ boson), and diboson ( $W W, Z Z, W Z$ ), and their contribution is estimated using simulated event samples normalised to the cross sections from NLO calculations as described in [2]. In order to estimate the dominant SM background contribution, a set of dedicated control regions (CRs) are defined for top and $W+$ jets backgrounds. The CRs are designed by applying the same selection as in SRs, except for two variables that are inverted. The 2J CRs are made orthogonal to the 2J SRs by inverting the cuts on $E_{\mathrm{T}}^{\mathrm{miss}}$ and $m_{\mathrm{T}}$. The $4 \mathrm{~J}$ and $6 \mathrm{~J} \mathrm{CRs}$ are made orthogonal to their corresponding SRs by inverting the cuts on $m_{\mathrm{T}}$ and aplanarity. The contributions from the major backgrounds are estimated by normalizing the predicted event yields from MC simulation to the number of data events observed in the dedicated CRs. The simulation is then used to extrapolate the background rates to the SRs by using a transfer factor $\left(\frac{N_{\mathrm{SR}}}{N_{\mathrm{CR}}}\right)$ approach. The extrapolation is then validated in dedicated Validation Regions (VRs) designed for each set of signal and control regions. Validation regions are typically defined such that they are kinematically closer to the signal regions, by inverting only one of the two discriminating variables used to make SRs orthogonal to the CRs. 


\section{Results and interpretation}

The results are interpreted in the aforementioned SUSY scenarios using a model-dependent fit. A simultaneous fit of the background and signal yields is performed across all $28 \mathrm{SR}$ and CR bins. The model-dependent fit is performed in order to set specific limits on the signal model. Figure 2 shows the $m_{\text {eff }}$ distributions in the $2 \mathrm{~J}$ bVeto and the $6 \mathrm{~J}$ bVeto regions after the fit. The dashed lines represent different benchmark signal samples. No significant excess over the SM background prediction is observed.

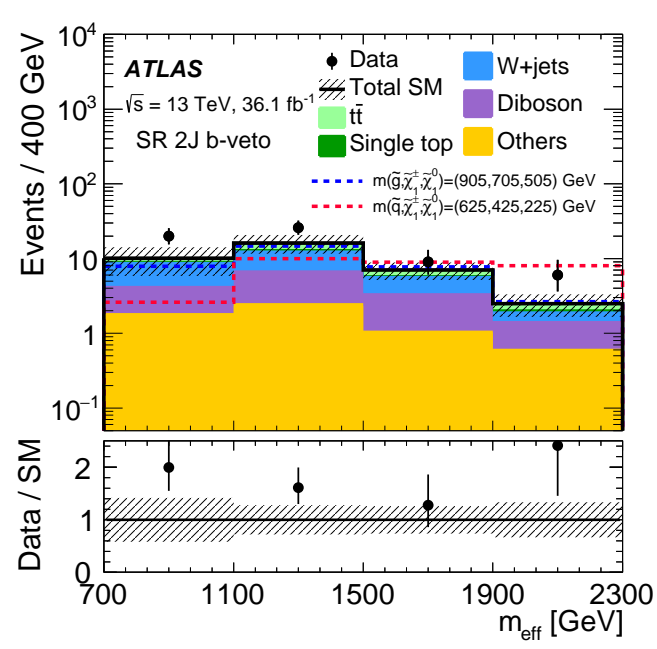

(a) SR 2J bVeto

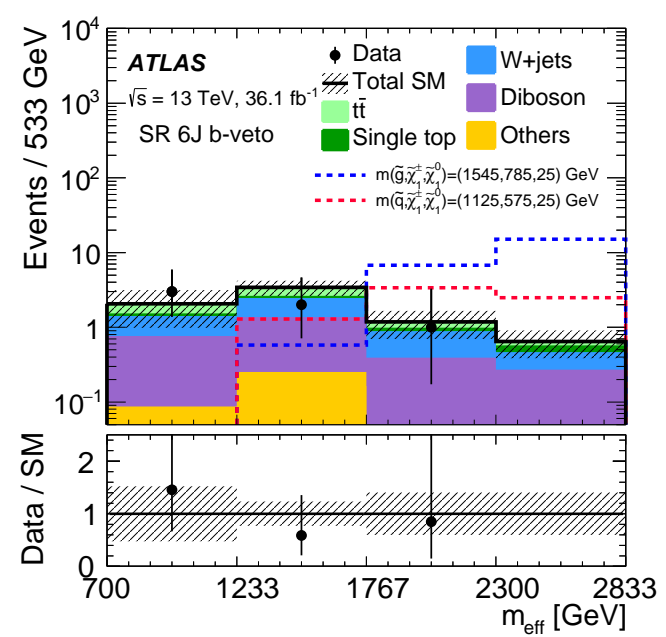

(b) SR 6J bVeto

Figure 2: The $m_{\text {eff }}$ distributions in (a) $2 \mathrm{~J}$, and (b) $6 \mathrm{~J}$ bVeto signal regions after fit [2].

Figure 3 shows the observed and expected exclusion limits at 95\% C.L. for the sparticle masses in different benchmark scenarios. The red solid line corresponds to the observed limit, and the dark gray dashed line indicates the expected limit with the $\pm 1 \sigma$ variation of the median expected limit indicated by the yellow band.

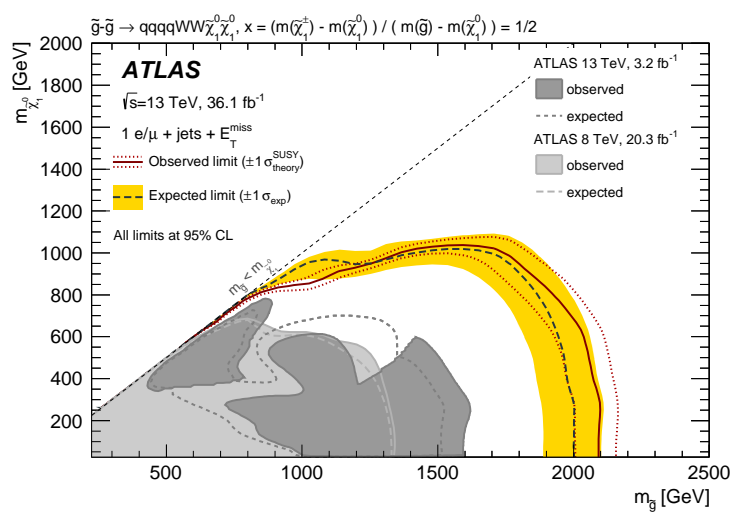

(a) gluino one-step $x=\frac{1}{2}$

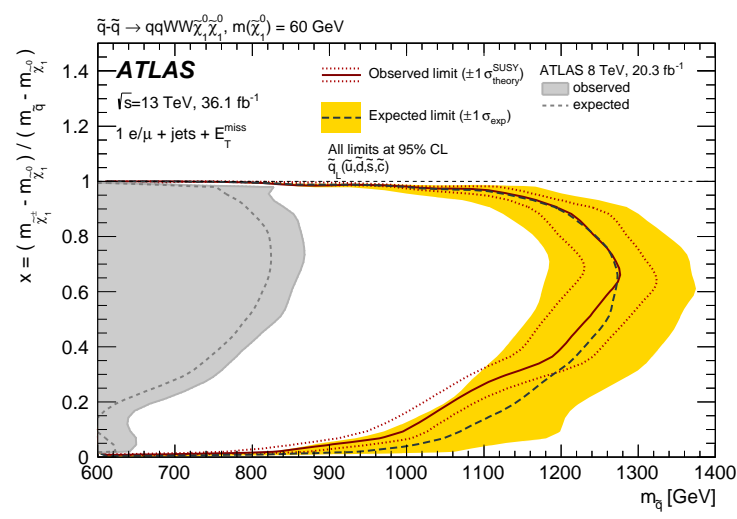

(b) squark one-step variable- $x$

Figure 3: Exclusion contours for (a) the gluino one-step $\left(x=\frac{1}{2}\right)$, and (b) the squark one-step (variable- $x$ ) [2]. 


\section{Conclusions}

The results of the search for gluino/squark pair production in final states with one isolated lepton, jets, and missing transverse energy have been presented for $36.1 \mathrm{fb}^{-1}$ data collected by the ATLAS detector at the LHC in 2015 and 2016. No significant excess over the Standard Model background prediction is observed. The exclusion limits have been extended up to $2.1 \mathrm{TeV}$ in gluino mass and $1.25 \mathrm{TeV}$ in squark mass.

\section{References}

[1] ATLAS Collaboration, The ATLAS Experiment at the CERN Large Hadron Collider, (2008) JINST 3 S08003

[2] ATLAS Collaboration, Search for squarks and gluinos in events with an isolated lepton, jets and missing transverse momentum at $\sqrt{s}=13 \mathrm{TeV}$ with the ATLAS detector, Phys. Rev D 96, 112010 (2017) 